Abstract P100 Table 1 Factors influencing reduced utilisation of healthcare services during the covid-19 pandemic

\begin{tabular}{|c|c|c|}
\hline & $\begin{array}{l}\text { Reduced use of healthcare } \\
\text { services due to the COVID- } \\
19 \text { pandemic/Total }\end{array}$ & Relative risk (RR) \\
\hline Total & $3333 / 8304(40.1 \%)$ & \\
\hline \multicolumn{3}{|l|}{ Respiratory comorbidities: } \\
\hline $\begin{array}{l}\text { One or more respiratory } \\
\text { comorbidities }\end{array}$ & $1388 / 2957(47.0 \%)$ & \multirow{2}{*}{$\begin{array}{c}\text { RR 1.29, } \\
p=<0.001 *\end{array}$} \\
\hline No respiratory comorbidity & $1945 / 5344$ (36.4\%) & \\
\hline \multicolumn{3}{|l|}{ Gender: } \\
\hline Male & $1823 / 4855$ (37.5\%) & \multirow{2}{*}{$\begin{array}{c}\text { RR 1.16, } \\
p=<0.001 *\end{array}$} \\
\hline Female & $1510 / 3446(43.8 \%)$ & \\
\hline \multicolumn{3}{|l|}{ Age: } \\
\hline $55-59$ & $545 / 1356(40.1 \%)$ & - \\
\hline $60-69$ & $1710 / 4216(40.6 \%)$ & RR $1.01 p=0.835$ \\
\hline $70-79$ & $1078 / 2729(39.5 \%)$ & RR $0.98 p=0.696$ \\
\hline \multicolumn{3}{|l|}{ Deprivation quintile: } \\
\hline Quintile 1 (most deprived) & $983 / 2474(39.7 \%)$ & - \\
\hline Quintile 2 & $932 / 2327(40.0 \%)$ & RR 1.01, $p=0.845$ \\
\hline Quintile 3 & $599 / 1510(39.7 \%)$ & RR 1.00, $p=0.995$ \\
\hline Quintile 4 & $606 / 1431(42.3 \%)$ & RR 1.07, $p=0.117$ \\
\hline Quintile 5 (least deprived) & $152 / 418(36.6 \%)$ & RR 0.92, $p=0.247$ \\
\hline
\end{tabular}

Results 8,304 participants completed a Year 1 lung health check between June 2020 and May 2021 (mean age 66.3, IQR 61-71, 4855 (58.5\%) male).

$3333(40 \%)$ reported reduced health service utilisation due to the COVID-19 pandemic. Of those, 3062 (91.9\%) stated this was due to the NHS cancelling or delaying appointments, whilst in 204 (6.1\%) appointments were cancelled by the participant. Reasons given by participants included not wanting to burden the NHS, difficulty with telephone consultations, and concern from media reports of hospital overcrowding.

Participants with a respiratory comorbidity (COPD, bronchiectasis, asthma, fibrosis, or sarcoidosis, as self-reported at initial study visit) (RR 1.29, $\mathrm{p}=<0.001$ ) and female participants ( $R R$ 1.16, $\mathrm{p}=<0.001$ ) were more likely to report impacted healthcare utilisation (table 1). Reported impact on healthcare use was not significantly affected by age or socioeconomic quintile.

Conclusions $40 \%$ of individuals in a cohort at high risk of lung cancer and respiratory comorbidities reported reduced utilisation of health care services due to the COVID-19 pandemic.

Limitations to our data include the lung health check questions not distinguishing between primary and secondary care or routine and urgent visits, and self-reported co-morbidity data limited to selected respiratory conditions. Nevertheless, we provide evidence for the scale of the problem and highlight that individuals with chronic respiratory conditions are particularly likely to be impacted.
Please refer to page A193 for declarations of interest related to this abstract.

\section{P101 MORTALITY IN PATIENTS REQUIRING HOME MECHANICAL VENTILATION DURING THE COVID-19 PANDEMIC: EXPERIENCES OF A REGIONAL SPECIALIST VENTILATION UNIT}

${ }^{1}$ RF D'Cruz, ${ }^{1} \mathrm{NM}$ Shah, ${ }^{2} \mathrm{~A}$ Learoyd, ${ }^{1} \mathrm{OJ}$ Elias, ${ }^{1} \mathrm{M}$ Mackie, ${ }^{1} \mathrm{~N}$ Weston, ${ }^{1} \mathrm{G}$ Kaltsakas, ${ }^{1}$ ES Suh, ${ }^{1} \mathrm{P}$ Marino, ${ }^{1} \mathrm{M}$ Ramsay, ${ }^{1} \mathrm{~S}$ Srivastava, ${ }^{1} \mathrm{H}$ Pattani, ${ }^{1} \mathrm{I}$ Steier, ${ }^{1} \mathrm{~N}$ Hart, ${ }^{1} \mathrm{~PB}$ Murphy. ${ }^{1}$ Lane Fox Respiratory Service, London, UK; ${ }^{2}$ School of Population Health and Environmental Sciences, King's College London, London, UK

\subsection{6/thorax-2021-BTSabstracts.210}

Introduction Deaths from severe acute respiratory syndrome coronavirus-2 (SARS-CoV-2) infection continue to increase in the UK. Patients with pre-existing comorbidities, including obesity and chronic respiratory disease, have been shown to be at increased risk of adverse clinical outcomes. The impact of COVID-19 on the survival of patients established on home mechanical ventilation (HMV) for chronic respiratory failure is currently unknown. We aimed to evaluate longitudinal trends in mortality amongst patients requiring HMV at a UK tertiary specialist ventilation unit.

Methods 20-year single-centre retrospective observational cohort study. Medical records of HMV users were used to determine mortality status and date of death. Primary cause of chronic hypercapnic respiratory failure was coded as chest 
wall disease, diseases of the lungs/airways, rapidly- or slowlyprogressive neuromuscular disease and obesity-related respiratory failure.

Results 4766 consecutive records between 1st January 2000 and 31st August 2020 were reviewed. Evaluation of deaths occurring during and subsequent to the UK second wave are ongoing. Mortality rates in March 2020 (1.68\%) and April 2020 (1.64\%) were higher than 2000-2019 (mean \pm SD $0.70 \pm 0.34 \%$ and $0.68 \pm 0.39 \%$ in March and April, respectively) (figure $1 \mathrm{~A}$ ), followed by a fall in May to $0.66 \%$, comparable to pre-COVID levels. A larger proportion of HMV users with obesity-related respiratory failure died between March-April 2020 (41.0\%) compared to the same period in 2019 (11.4\%) and all previous years (figure 1B). The proportion of HMV users with lung/airways disease, slowly-progressive neuromuscular disease and chest wall disease who died in this period were reduced compared to previous years.
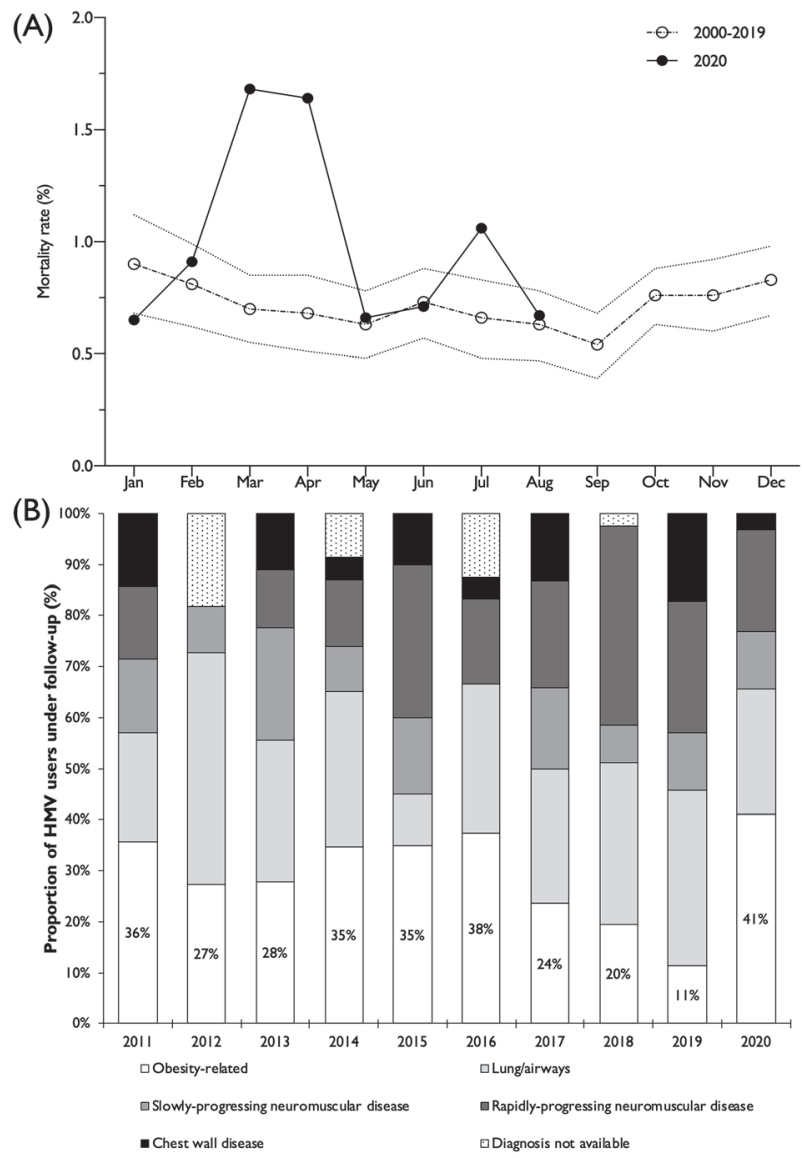

Abstract P101 Figure 1 (A) Monthly mortality of patients under Lane Fox Respiratory Service follow-up, dotted lines represent upper and lower bounds of $95 \%$ confidence intervals (B) Proportion of home mechanical ventilation (HMV) users in each disease category who died between 1st March and 30th April by year

Conclusions Deaths amongst HMV users at our regional ventilation centre were highest in the first two months following the onset of the COVID-19 pandemic. A subsequent fall in mortality may relate to effective shielding advice following national lockdown and departmental guidance offered. The majority of deaths were in patients with obesity-related respiratory failure. These data support previous observations that obesity is a major risk factor for adverse outcomes in patients with COVID-19.

\section{P102 PSYCHOSOCIAL THEMES OF THE IMPACT OF THE COVID-19 PANDEMIC AND SHIELDING IN ADULTS AND CHILDREN WITH EARLY-ONSET NEUROMUSCULAR DISORDERS AND THEIR FAMILIES}

L Spurr, H-L Tan, R Wakeman, M Chatwin, A Simonds. Royal Brompton Hospital, London, UK

\subsection{6/thorax-2021-BTSabstracts.211}

Introduction Most patients with early-onset neuromuscular disorders (NMDs) were advised to shield during the pandemic due to assumptions that treatments e.g. corticosteroids, or complications e.g. pre-existing respiratory failure would increase COVID-19 risk. It remains poorly understood how those with NMDs and their families approached and responded to risk mitigating strategies e.g. shielding. We aimed to determine themes describing the psychosocial impact of the COVID-19 pandemic and measures taken to reduce risk in this population.

Methods In-depth questionnaires specifically designed to meet research aims were completed by telephone between September 17 th and December 31st 2020 by patients with NMDs or their parent. Inductive thematic analysis was performed to first code transcriptions of audio recordings of questionnaires then develop candidate themes by exploring coded data. Candidate themes were evaluated against the original data set before defining final themes: participant validation was sought to provide additional confirmation of accuracy.

Results 40 questionnaires were completed: patients were $70 \%$ male, aged 2 to 48 years with NMDs e.g. muscular dystrophies, spinal muscular atrophy. $80 \%$ required long-term noninvasive or tracheostomy ventilation. Three themes were identified: 1) concern regarding the health impact of COVID-19; 2) perceptions of strategies to prevent SARS-CoV-2 transmission; 3) psychological impact of the COVID-19 pandemic. Anxiety, fear and worry were the most frequently reported emotions, particularly in relation to health risk of COVID-19, but level and pervasiveness fluctuated during the pandemic. Strict adherence to shielding was reported at the start of the pandemic but was often relaxed due to 1) official guidance, 2) emerging evidence of less severe outcomes in children and NMD cohorts, and 3) unsustainability of limited social contact including cessation or reduction in personal care. Concern about hospital attendance during the pandemic, and anxiety regarding perceived lack of access to Intensive Care were common.

Conclusions Measures to reduce transmission of COVID-19 have disproportionally affected patients with NMDs and their families. For most, negative psychosocial impacts have and will continue to improve, particularly due to the success of the vaccination programme. These aspects should be considered when advising patients and families on risk and risk-mitigating strategies during the current and future pandemics. 